This item was submitted to Loughborough's Research Repository by the author.

Items in Figshare are protected by copyright, with all rights reserved, unless otherwise indicated.

\title{
The influence of touchdown conditions and contact phase technique on post- flight height in the straight handspring somersault vault
}

PLEASE CITE THE PUBLISHED VERSION

http://dx.doi.org/10.1016/j.jbiomech.2014.06.020

PUBLISHER

(C) Elsevier Ltd.

VERSION

AM (Accepted Manuscript)

\section{PUBLISHER STATEMENT}

This work is made available according to the conditions of the Creative Commons Attribution 3.0 International (CC BY 3.0) licence. Full details of this licence are available at: https://creativecommons.org/licenses/by/3.0/

\section{LICENCE}

CC BY 3.0

\section{REPOSITORY RECORD}

Yeadon, Fred, Monique I. Jackson, and Michael Hiley. 2014. "The Influence of Touchdown Conditions and Contact Phase Technique on Post-flight Height in the Straight Handspring Somersault Vault". Loughborough University. https://hdl.handle.net/2134/16413. 
Journal of Biomechanics 47, 3143-3148, 2014

\title{
The influence of touchdown conditions and contact phase technique on post-flight height in the straight handspring somersault vault
}

\author{
Maurice R. Yeadon, Monique I. Jackson and Michael J. Hiley
}

School of Sport, Exercise and Health Sciences, Loughborough University, Loughborough LE11 3TU, UK.

\begin{abstract}
In vaulting the gymnast must generate sufficient linear and angular momentum during the approach and table contact in order to complete the rotational requirements in the postflight phase. This study investigated the effects of touchdown conditions and contact technique on peak post-flight height of a straight handspring somersault vault. A planar seven-segment torque-driven computer simulation model of the contact phase in vaulting was evaluated by varying joint torque activation time histories to match three performances of a straight handspring somersault vault by an elite gymnast. The closest matching simulation was used as a starting point to optimise peak post-flight height of the mass centre for a straight handspring somersault. It was found that optimising either the touchdown conditions or the contact technique increased post-flight height by $0.1 \mathrm{~m}$ whereas optimising both together increased post-flight height by $0.4 \mathrm{~m}$ above that of a simulation matching the recorded performance. Thus touchdown technique and contact technique make similar contributions to post-flight height in the straight handspring somersault vault. Increasing touchdown velocity and angular momentum lead to additional post-flight height although there was a critical value of vertical touchdown velocity beyond which post-flight height decreased.
\end{abstract}

\section{INTRODUCTION}

The mechanics of the table contact phase of gymnastics vaulting is dependent on the initial contact conditions and the technique used during the contact phase. Although there are numerous coaching publications on vaulting, there is no general consensus about the importance of table contact phase technique. Some authors hold the view that vaulting performance is primarily determined prior to table contact (Still, 1990) while others have suggested that the gymnast has the ability to change the outcome of the vault via table contact technique (Smith, 1982; Boone, 1976).

Takei (1988) used correlational analysis to show that a large pre-flight horizontal velocity is an important determinant of success in the tucked handspring somersault vault. Additionally Takei and Kim (1990) found that a large change in mass centre vertical velocity during contact was beneficial. While the horizontal pre-flight velocity is clearly an aspect of touchdown conditions, it is unclear whether the change in vertical velocity is a function of initial conditions or contact technique or both.

King et al. (1999) used a passive (no shoulder torque) two-segment simulation model to determine optimum touchdown conditions for a straight handspring somersault vault (which continues the pre-flight rotation, Figure 1a) and a Hecht vault (which reverses the pre-flight rotation during contact, Figure 1b). It was found that quite different touchdown conditions were required for the two vaults and were similar to those used in actual performances, indicating that initial conditions can have a profound effect. King and Yeadon (2005) subsequently used a five-segment torquedriven model to simulate the Hecht vault and found that shoulder torque during the contact phase had only a small effect on post-flight performance. 

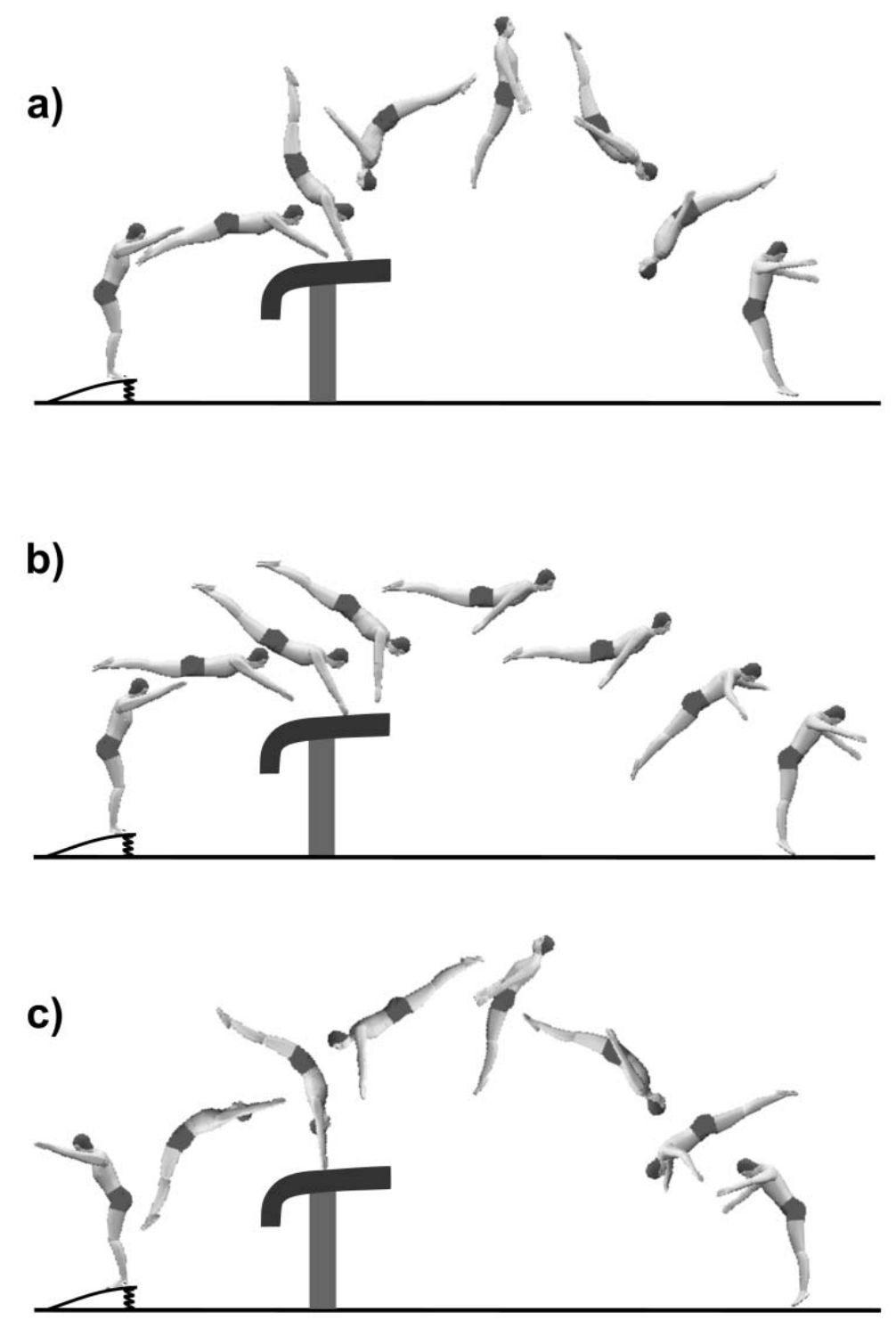

Figure 1. The (a) straight handspring somersault, (b) Hecht and (c) straight Yurchencho somersault vaults.

Koh et al. (2003) used a five-segment angle-driven simulation model with joint torque constraints to optimise the post-flight of a straight Yurchenko vault (backward entry, Figure 1c). The optimum simulation had similar post-flight horizontal velocity to the actual performance but greater vertical velocity at takeoff resulting in greater peak height in post-flight. The optimal vault was achieved by modifying the initial conditions (after impact) and contact phase technique. The relative importance of these two contributors to improved performance in vaults that continue the pre-flight rotation (Figure 1a, c) remains unknown. The straight handspring somersault vault (Figure 1a) forms the basis of the 12 most difficult forward entry vaults seen in elite male competition (FIG, 2013).

The aims of this study were to investigate the effect on peak post-flight height of the straight handspring somersault vault arising from (a) initial conditions at touchdown and (b) joint torques during the contact phase (technique). 


\section{METHODS}

In order to investigate the effects of initial conditions and table contact technique on vaulting performance a torque-driven computer simulation model was developed. Subsections in methods describe data collection on an elite gymnast, the simulation model, the method of model evaluation, and the application of the model.

\section{Data Collection}

An internationally competitive male gymnast (21 years, $69.9 \mathrm{~kg}, 1.73 \mathrm{~m}$ ) gave informed consent to perform six straight handspring somersault vaults (Figure 1a). Eighteen Vicon MX13 cameras, sampling at $480 \mathrm{~Hz}$, were used to track the motion of markers attached to the gymnast and the vaulting table. An international Brevet judge assessed and ranked the performance of each vault, with the best three vaults selected for subsequent analysis. Details of the methods used to determine the kinematics of the performances are given in Jackson et al. (2011).

Gymnast-specific torque parameters were calculated from measurements taken from the gymnast during maximal voluntary contractions using a Con-trex isovelocity dynamometer. Data were obtained for extension and flexion of the wrist, shoulder, hip and knee at angular velocities ranging from $30^{\circ} \mathrm{s}^{-1}$ to $400^{\circ} \mathrm{s}^{-1}$. Torque surfaces were fit to the data based on the relationships between torque, angle and angular velocity as detailed in Forrester et al. (2011). Passive torque data were also obtained using the isovelocity dynamometer for shoulder flexion, wrist extension and hip extension. Exponential equations were fit to the passive torque-angle data for each joint (Riener and Edrich, 1999; Esteki and Mansour, 1996).

Ninety-five anthropometric measurements were taken from the gymnast and gymnast-specific segmental inertia parameters were calculated using the model of Yeadon (1990a). The mass and dimensions of the vaulting table were measured and the inertial parameters calculated as in Jackson et al. (2011).

\section{Simulation Model}

A two-dimensional torque-driven simulation model of gymnastics vaulting was developed using the software package Autolev ${ }^{\mathrm{TM}}$. A planar model was used since non-twisting vaults are essentially symmetrical about the sagittal plane. The model simulated the interaction between a seven-segment gymnast and a single-segment vaulting table during the table contact phase of the vault.

The gymnast was modelled using seven rigid segments to represent the fingers, the palms, the arms, the head + upper trunk, the lower trunk, the thighs and the shanks (Figure 2). A damped linear spring was used to represent shoulder retraction and protraction, whilst displacement of the glenohumeral joint centre was modelled as a cubic function of the shoulder angle as in Begon et al. (2008). A damped torsional spring was used to represent hand flexion/extension at the knuckles, whilst flexion/extension of the trunk was modelled as a function of the hip angle as in Yeadon (1990b). A non-linear, damped torsional spring allowed the table to rotate about its centre of rotation.

The interaction between the gymnast and the table was modelled as detailed in Jackson et al. (2011). The normal contact force was represented by spring-dampers situated at three points of contact: the fingertip, the knuckle and the base of the palm, while the tangential contact force was modelled using a two-state frictional force representation to allow for both static and dynamic friction. 


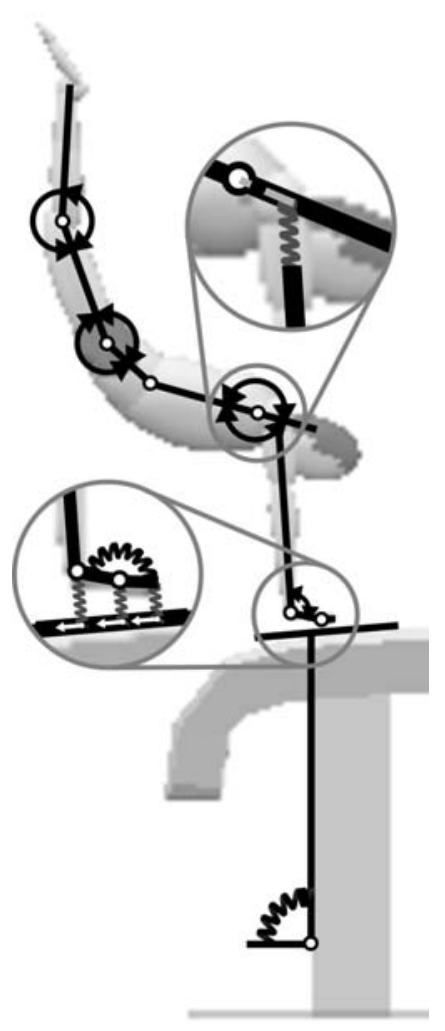

Figure 2. Vaulting table contact phase simulation model

The model was driven by torque generators, consisting of contractile and series elastic components, which acted to extend and flex the wrist, shoulder, hip and knee. The torque generators were defined based on the measured torque-angle-angular velocity relations and represented the maximum voluntary torques that the gymnast could produce.

To determine the applied torque the maximal torque was multiplied by an activation level lying between 0 and 1. A quintic function (Hiley and Yeadon, 2003) was used to ramp up/down the activation level. The extensors were allowed to ramp up from an initial level $(<0.5)$ and ramp down towards the end of the simulation. In contrast the flexors were allowed to ramp down from an initial level and then ramp up towards the end of the simulation to prevent hyper-extension (King et al., 2009). The shoulder flexor was regarded as an extensor since it was responsible for increasing the shoulder joint angle and the shoulder extensor was regarded as a flexor. Seven parameters were required to specify the timing and level of activation for each torque generator (two start times, two ramp durations, three activation levels), giving a total of 56 parameters. In addition to the active torque generators, passive torque elements, based on the measured exponential torque-angle relations, were included at the wrist, shoulder and hip joints.

The model parameters (viscoelastic parameters of the shoulder, knuckle, table and contact springs and the static and kinetic coefficients of friction between the hands and the contact surface) were set to those determined in Jackson et al. (2011) apart from the damping parameter of the contact spring, which was increased to $10,000 \mathrm{Nm}^{-1} \mathrm{~s}$ to prevent the hands from 'bouncing' during the initial part of the table contact phase. The input to the torque-driven model comprised the initial conditions at contact (joint angles and velocities, upper trunk angle and velocity, mass centre position and velocity) together with the activation time histories of the torque 
generators. The output from the torque-driven model comprised the joint angles and the orientation of the upper trunk, the mass centre position and velocity, and the angular momentum about the mass centre.

\section{Model Evaluation}

To ensure that the torque-driven simulation model produced realistic human movements, an evaluation of the model was conducted by assessing how accurately simulations matched the recorded performances of the three vaults. A genetic algorithm (Carroll, 1996) varied the torque generator activation parameters plus three initial conditions (gymnast's centre of mass horizontal and vertical velocity and upper trunk angular velocity) to minimise an objective function score that measured the difference between a simulation and a recorded performance. The initial linear and angular velocities were allowed to vary from the measured values by $\pm 0.1 \mathrm{~ms}^{-1}$ and \pm 0.5 rads $^{-1}$ respectively in order to compensate for reconstruction errors in the kinematic data.

The objective difference score was composed of four performance components $\left(P_{i}\right)$, and eight configuration components $\left(C_{i}\right): P_{1}$ - difference in upper trunk orientation at take-off $\left({ }^{\circ}\right), \mathrm{P}_{2}-\%$ difference in angular momentum at take-off, $\mathrm{P}_{3}-$ $\%$ difference in horizontal linear velocity at take-off, $\mathrm{P}_{4}-\%$ difference in vertical linear velocity at take-off, $\mathrm{C}_{1}$ - Root mean square (RMS) difference in wrist angle during contact $\left(^{\circ}\right), \mathrm{C}_{2}$ - RMS difference in shoulder angle during contact $\left(^{\circ}\right), \mathrm{C}_{3}-\mathrm{RMS}$ difference in hip angle during contact $\left(^{\circ}\right), \mathrm{C}_{4}$ - RMS difference in knee angle during contact $\left({ }^{\circ}\right), \mathrm{C}_{5}$ - difference in wrist angle at take-off $\left(^{\circ}\right), \mathrm{C}_{6}$ - difference in shoulder angle at take-off $\left({ }^{\circ}\right), \mathrm{C}_{7}$ - difference in hip angle at take-off $\left(^{\circ}\right), \mathrm{C}_{8}$ - difference in knee angle at take-off $\left({ }^{\circ}\right)$. The overall difference score for the simulation was calculated by taking the RMS of the twelve components. The performance and configuration categories were each given a $50 \%$ weighting and within each category the components were equally weighted, where $1^{\circ}$ was considered comparable to a $1 \%$ difference in other measures (Equation 1):

$$
\text { Score }=\sqrt{\left(\frac{P_{1}^{2}+P_{2}^{2}+P_{3}^{2}+P_{4}^{2}}{8}\right)+\left(\frac{C_{1}^{2}+C_{2}^{2}+C_{3}^{2}+C_{4}^{2}+C_{5}^{2}+C_{6}^{2}+C_{7}^{2}+C_{8}^{2}}{16}\right)}
$$

Penalties were imposed if the joints exceeded anatomical limits.

\section{Optimisation}

The simulation model was used to optimise post-flight height in a straight handspring somersault vault by manipulating the initial conditions and table contact technique. The procedure used a parallelised genetic algorithm (Soest and Casius, 2003) that was run on a high performance computer with 60 processors. In all optimisations the recommended optimisation algorithm tuning was used except for the selection of single point crossovers (Carrol, 1996). Single point crossovers were chosen as this was deemed appropriate for the application, since sections of activation profiles could then be passed on to the offspring rather than just single parameter values. Penalties were used to ensure the model had an appropriate time of flight and sufficient rotation potential to complete the vault. Rotation potential is the number of straight somersaults (with arms adducted) that could be performed in post-flight (Hiley and Yeadon, 2008). 
The post-flight time was defined as the time from the last instant of take-off from the table until the first instant of landing. It was assumed that the gymnast had a touchdown angle at landing of $35^{\circ}$ short of vertical, as the angular momentum at landing would cause the gymnast to rotate forwards about the feet during the initial part of the landing phase (King et al., 1999). The corresponding height of the mass centre of the model at landing was calculated to be $0.74 \mathrm{~m}$ and was used in all optimisation simulations for consistency. The peak post-flight height and time $t_{p f}$ were then calculated using constant acceleration equations.

The calculation of rotation potential took into account the body orientation at take-off from the vaulting table, as well as the angular momentum at take-off and the post-flight time. The body orientation angle $\phi_{\text {to }}$ at take-off was calculated from the relative positions of the fingers and the centre of mass at take-off. The rotation potential angle $\phi_{p f}$ for post-flight was calculated as the product of the angular momentum $H$ about the mass centre at take-off and the post-flight time $t_{p f}$, divided by the moment of inertia $I_{c m, s}$ about the centre of mass of the gymnast in a straight position with adducted arms $\left(10.7 \mathrm{~kg} \cdot \mathrm{m}^{2}\right)$. The rotation potential $\phi$ was the sum of the body orientation angle at take-off $\phi_{t o}$ and the post-flight phase rotation potential $\phi_{p f}$ expressed in straight somersaults.

Three optimisations were carried out in order to maximise the post-flight height under different conditions. In the first optimisation the table touchdown configuration (shoulder and hip angle) and orientation (torso angle) were varied whilst maintaining the pre-flight parabola and angular momentum of the recorded performance. The shoulder angle was allowed to vary from $90^{\circ}$ to $190^{\circ}$, the hip angle from $30^{\circ}$ of flexion to $30^{\circ}$ of hyperextension and the torso angle from $30^{\circ}$ below to $50^{\circ}$ above the horizontal. In the first optimisation the torque generator activations obtained from the matching simulation were used. The second optimisation used the touchdown configuration and orientation from the recorded performance and the torque generator activation parameters were varied in order to determine the potential improvement arising from contact phase technique changes for the same touchdown conditions. Finally in the third optimisation both the configuration and torque generator activation parameters were allowed to vary in order to maximise post-flight height. Again the model was constrained to stay on the parabola defined by the gymnast's horizontal and vertical velocity at springboard take-off.

Additionally three sets of simulations were run, assuming the same contact phase technique and touchdown configuration as in the third optimisation, but with increasing horizontal and vertical touchdown velocities and angular momentum about the mass centre in order to determine the effect of changes in touchdown conditions. All three touchdown parameters were increased to $130 \%$ of the touchdown values in the third optimisation.

\section{RESULTS}

\section{Model evaluation}

Evaluation of the torque-driven model showed close correspondence with the performance data (Figure 3a, b). Overall difference scores of 3.0\%, 2.0\% and 3.5\% were obtained for the three vaults considered, demonstrating that the torque-driven model was capable of replicating vaulting performance and therefore was suited for subsequent performance optimisation. The results were comparable for the three trials so only one trial was used for the post-flight height optimisation analysis. The 
closest matching simulation was selected for this purpose and had a peak post-flight mass centre height of $2.72 \mathrm{~m}$ compared with $2.71 \mathrm{~m}$ for the performance.

\section{Optimisation}

The peak post-flight height of the matching simulation was $2.72 \mathrm{~m}$. When the configuration and orientation at touchdown were varied (optimisation 1) the peak post-flight height increased to $2.83 \mathrm{~m}$ (Figure 3c). When the torque generator activation parameters were varied (optimisation 2), while maintaining the recorded touchdown configuration and orientation, the peak post-flight height was $2.82 \mathrm{~m}$ (Figure 3d). In the third optimisation where the touchdown configuration and orientation along with the table contact activations were allowed to vary (optimisation 3) the peak post-flight height increased to $3.12 \mathrm{~m}$ (Table 1, Figure 3e).

Table 1. Table touchdown and take-off variables from the matching and optimised simulations

\begin{tabular}{|c|c|c|c|c|}
\hline Simulation & Matchin & Opt1 & Opt2 ${ }^{+}$ & Opt3 \\
\hline \multicolumn{5}{|l|}{ Touchdown angle! } \\
\hline torso $\left[{ }^{\circ}\right]$ & $5^{\circ}$ & $-21^{c}$ & $5^{\circ}$ & $-33^{\circ}$ \\
\hline shoulder $\left[{ }^{\circ}\right]$ & $134^{\circ}$ & $102^{\circ}$ & $134^{\circ}$ & $97^{\circ}$ \\
\hline hip $\left[^{\circ}\right]$ & $187^{\circ}$ & $204^{\circ}$ & $187^{\circ}$ & $202^{\circ}$ \\
\hline \multicolumn{5}{|l|}{ Touchdown CoM } \\
\hline ver. position [m] & 1.81 & 1.77 & 1.81 & 1.62 \\
\hline ver. velocity $\left[\mathrm{ms}^{-1}\right]$ & 2.83 & 2.96 & 2.82 & 3.41 \\
\hline hor. velocity $\left[\mathrm{ms}^{-1}\right]$ & 5.10 & 5.10 & 5.10 & 5.10 \\
\hline \multicolumn{5}{|l|}{ Take-off angles } \\
\hline torso $\left[{ }^{\circ}\right]$ & $76^{\circ}$ & $79^{\circ}$ & $85^{\circ}$ & $91^{\circ}$ \\
\hline shoulder $\left[{ }^{\circ}\right]$ & $181^{\circ}$ & $167^{\circ}$ & $191^{\circ}$ & $185^{\circ}$ \\
\hline hip $\left[^{\circ}\right]$ & $199^{\circ}$ & $196^{\circ}$ & $192^{\circ}$ & $178^{\circ}$ \\
\hline \multicolumn{5}{|l|}{ Take-off CoM } \\
\hline ver. position [m] & 2.33 & 2.34 & 2.38 & 2.43 \\
\hline ver. velocity $\left[\mathrm{ms}^{-1}\right]$ & 2.77 & 3.08 & 2.94 & 3.72 \\
\hline hor. velocity $\left[\mathrm{ms}^{-1}\right]$ & 3.88 & 4.12 & 3.88 & 3.53 \\
\hline \multicolumn{5}{|l|}{ Post-flight } \\
\hline peak height [m] & 2.72 & 2.83 & 2.82 & 3.13 \\
\hline rot. potential [ss] & 1.37 & 1.34 & 1.34 & 1.35 \\
\hline time [s] & 0.92 & 0.97 & 0.95 & 1.08 \\
\hline
\end{tabular}

* with the activations from the matching simulation

+ with the initial configuration and orientation from the matching simulation 

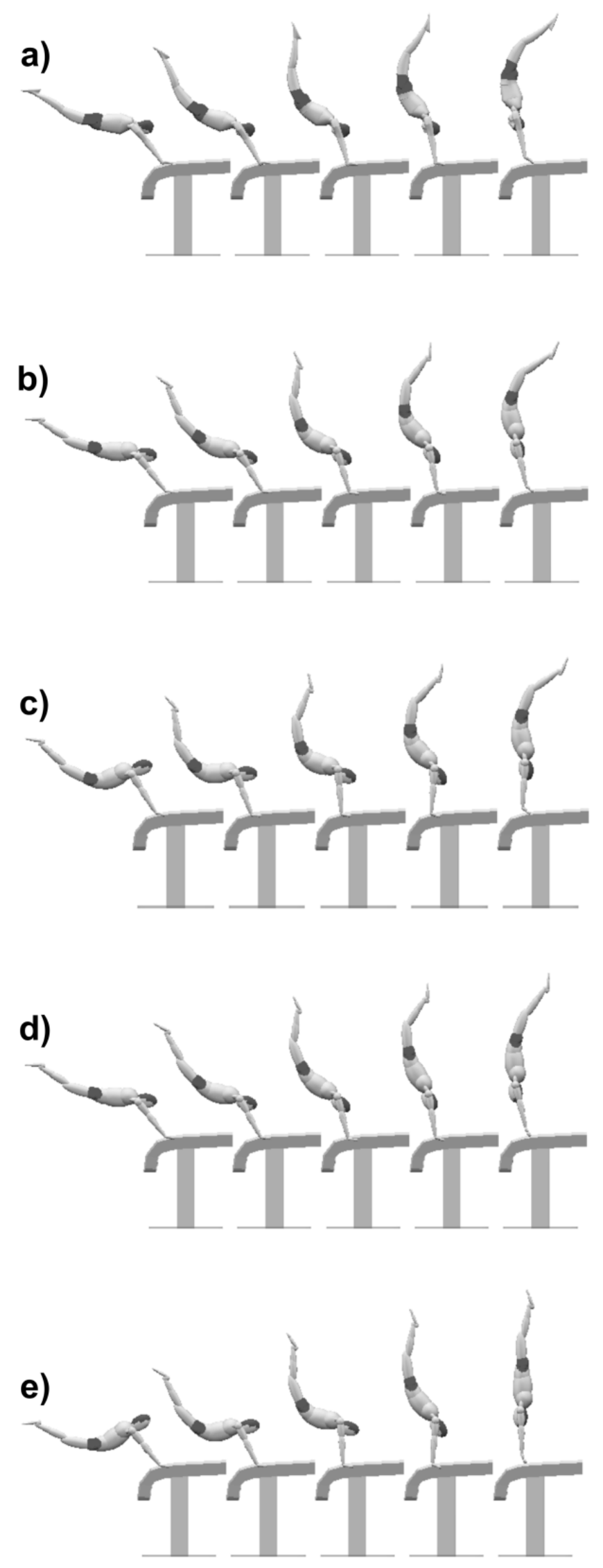

Figure.3. Simulated vaulting performances: (a) recorded performance, (b) matching simulation, (c) optimised touchdown configuration, (d) optimised contact phase technique, and (e) optimised touchdown configuration and contact phase. 
The hip and shoulder torques were generally greater in optimisations 2 and 3 than in the matching simulation (Figure 4).
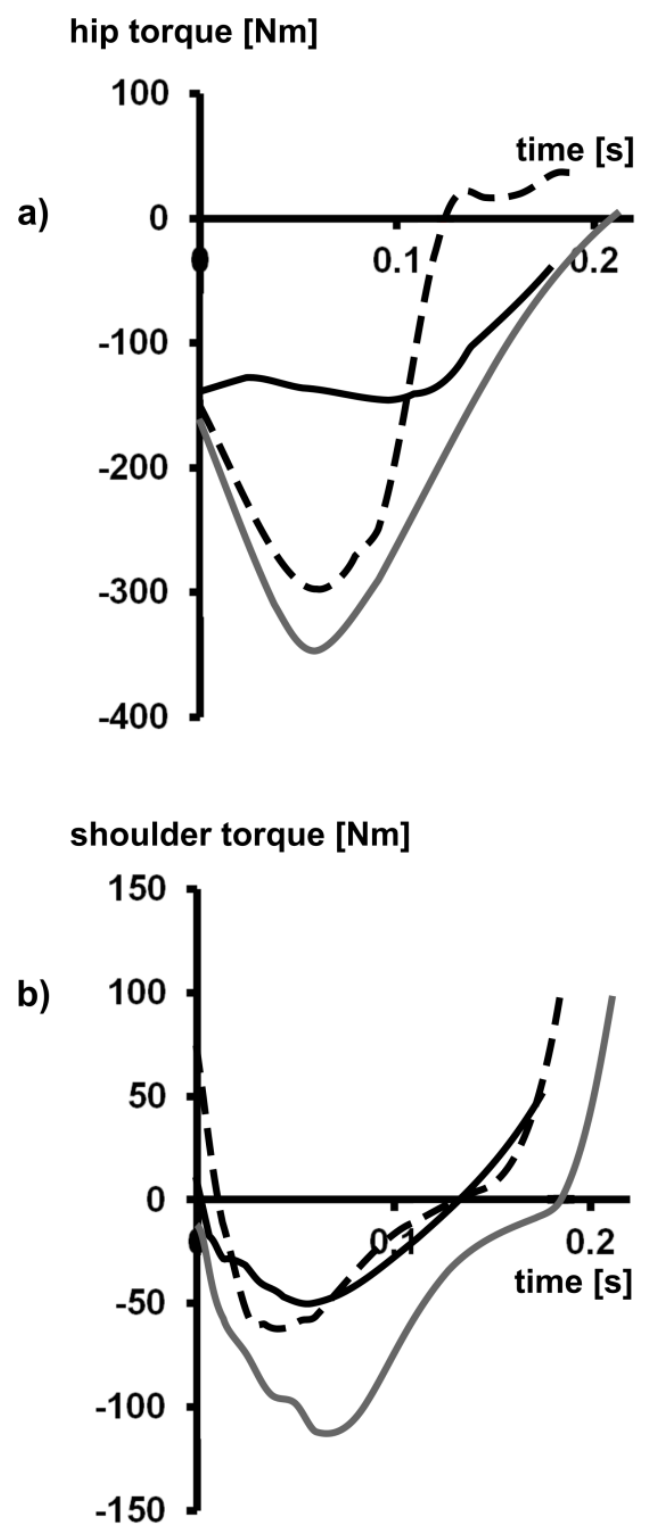

Figure 4. Time histories of (a) hip torque and (b) shoulder torque for matching simulation (solid black), optimisation 2 (dashed) and optimisation 3 (solid grey).

When the touchdown horizontal and vertical velocities and angular momentum were independently increased post-flight height and rotation potential were affected (Figures 5,6). As horizontal touchdown velocity increased both post-flight height and rotation potential increased (Figures $5 \mathrm{a}, 6 \mathrm{a}$ ). However, there appeared to be a plateau in the peak post flight height (Figure 5a). As the vertical velocity at touchdown was increased post-flight height and rotation potential both increased initially, but then decreased (Figures 5b, 6b). As angular momentum at touchdown was increased post-flight height and rotation potential both increased (Figures $5 \mathrm{c}$, 6c). 

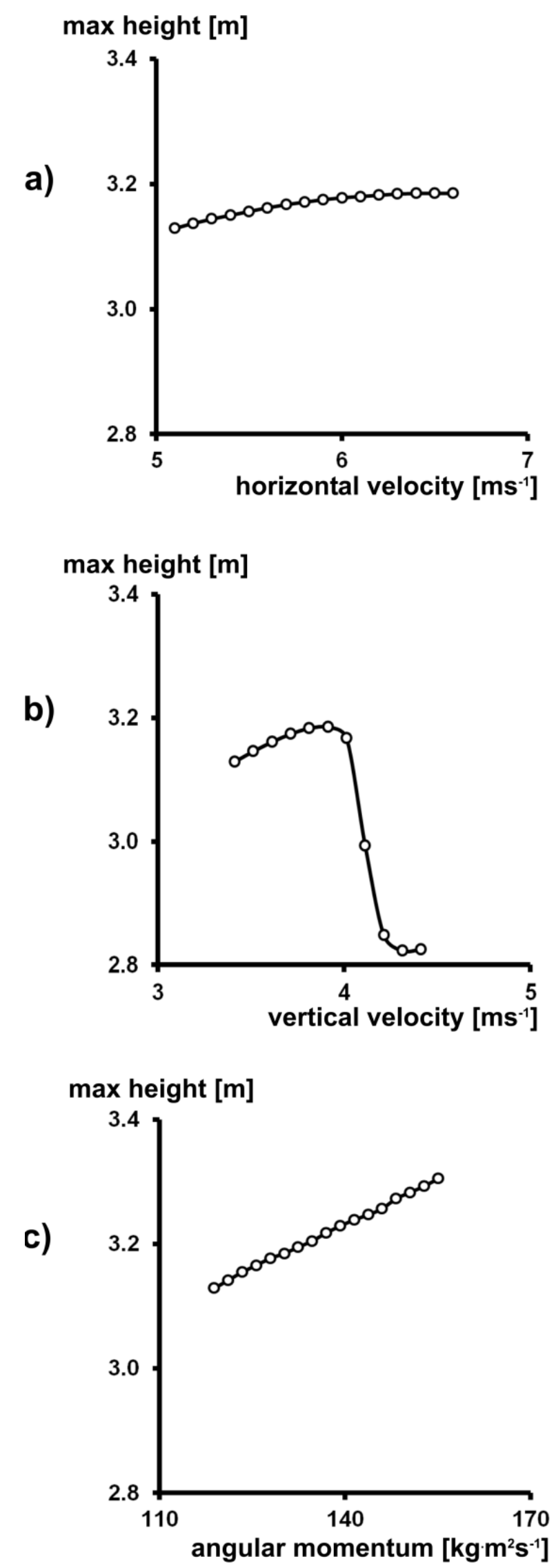

Figure 5. Changes in maximum height in flight when varying (a) touchdown horizontal velocity, (b) vertical velocity and (c) angular momentum respectively. 

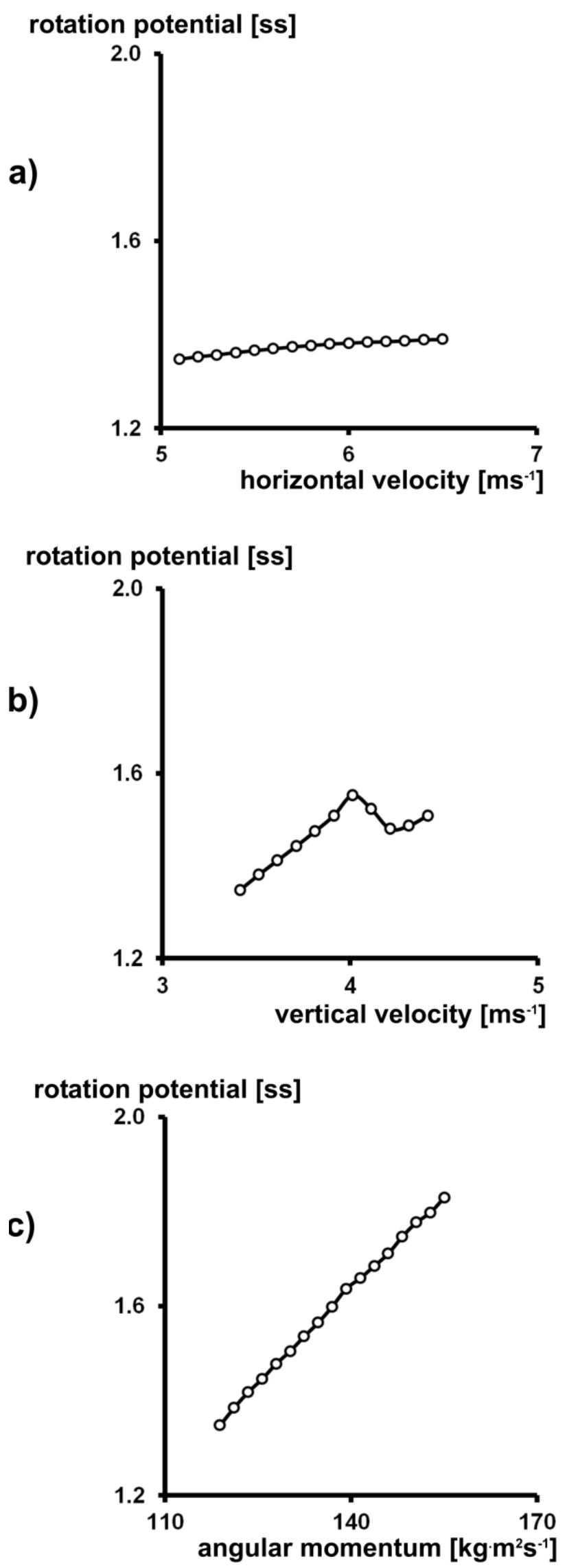

Figure 6. Changes in rotation potential (measured in straight somersaults, ss) when varying touchdown (a) horizontal velocity, (b) vertical velocity and (c) angular momentum respectively. 


\section{DISCUSSION}

The aim of this study was to investigate the extent to which the conditions at vaulting table touchdown and the technique adopted during table contact affected peak post-flight height during a straight handspring somersault vault.

When the initial configuration and orientation of the model were allowed to vary (optimisation 1), an increase in peak post flight height of $0.11 \mathrm{~m}$ was achieved compared to the matching simulation (Table 1). During the optimisation the activations obtained from the matching simulation were maintained. When only the technique during table contact was allowed to vary (optimisation 2), the increase in peak post flight height was $0.10 \mathrm{~m}$ above the matching simulation. When the configuration and orientation were allowed to vary along with the joint torque activation parameters (optimisation 3 ) the optimal peak height in post-flight increased by $0.41 \mathrm{~m}$. These results suggest that changes in initial conditions and contact technique have a similar influence on post-flight height.

Optimal configuration results in a lower centre of mass position at contact so that the gymnast has a shorter pre-flight and contacts the vault earlier on the preflight parabola (Figure 3). As the simulation constrains the vault to remain on the same pre-flight parabola this earlier contact results in a greater upwards vertical touchdown velocity. Optimal configuration is less stretched out, which gives a smaller moment of inertia and consequently angular velocity increases.

The hip and shoulder torques in optimisations 2 and 3 were generally greater than in the matching simulation (Figure 4). Shoulder torque allows the shoulder angle to open thereby doing work and raising the mass centre. If the shoulder torque is increased there will be a tendency for the hips to flex and so the hip extensor torque must also increase. All torques were within the capability of the gymnast as determined from dynamometer measurements.

In all three optimisations the model was constrained during pre-flight so that the mass centre lay on the parabola defined by the gymnast's horizontal and vertical velocity from the recorded performance. In addition the angular momentum about the mass centre was constrained to be the same as in the recorded performance. It may have been possible for the gymnast to increase both his pre-flight velocity and angular momentum. However, since there were no data on the limits and the interaction between these parameters, they were maintained at the measured values.

To investigate the effect of the touchdown velocity and the angular momentum on post-flight height, these variables were independently increased systematically up to $130 \%$ of the optimisation 3 values. In all cases increasing horizontal and vertical velocity and angular momentum lead to an initial increase in peak post-flight height (Figure 5). Increasing pre-flight angular momentum had the largest effect on peak post-flight height. Increasing horizontal and vertical velocity at touchdown lead to an increase in peak post-flight height, but only up to a point (Figures 5a, b). The drop observed in post-flight height when increasing the vertical velocity was associated with a drop in table contact time and an insufficient effect of the table contact technique. As the pre-flight vertical velocity increased beyond a critical point the model was unable to do as much work during the table contact phase. The effect of increasing horizontal velocity was less marked. However, within the ranges used, it appears that greater pre-flight angular momentum leads to greater peak post-flight height (Figure 5c).

The straight handspring somersault vault has previously been optimised for both post-flight height and distance using a passive two-segment simulation model 
(King et al., 1999). The optimal configuration during the instantaneous table contact was found to be with $178^{\circ}$ between arms and torso. In the recorded performance and the optimisations that varied the initial configuration and orientation (optimisations 1 and 3), the configuration includes a closed shoulder angle and varying degrees of hip hyperextension (Figure 3 , Table 1 ). These differences may be accounted for by the simplicity of the two-segment model which assumed instantaneous table contact and zero shoulder torque. In the current model work is able to be done by shoulder torque in opening the shoulder angle during contact.

While the 5-segment vaulting model of King and Yeadon (2005) showed that shoulder torque had only a small effect on post-flight performance in the Hecht vault, this is not the case for the straight handspring somersault as shown in the present study. The difference is that the initial conditions of the Hecht vault produce a passive and rapid closing of the shoulder angle so that the shoulder torque available to accelerate this process is limited due to the high concentric conditions. In the straight handspring somersault vault any such tendency for the shoulder angle to close will put the shoulder torque that opens the shoulder angle into eccentric conditions which will make high torques available. As a consequence hip and shoulder torques are able to do substantial work during the contact phase resulting in greater peak post-flight height.

The increase in height of $0.4 \mathrm{~m}$ between performance $(2.7 \mathrm{~m})$ and optimum simulation $(3.1 \mathrm{~m})$ may seem rather high but is consistent with the heights reached in elite performances of handspring double front somersault (Roche) vaults $(3.0 \pm 0.1$ $\mathrm{m}$ ) which have a similar angular momentum requirement (Takei, 2007). This suggests that the improvement is feasible.

The simulations presented here demonstrate that changes in initial conditions and contact technique have a similar influence on post-flight height. Increasing horizontal approach velocity will improve performance in agreement with Takei (1988). However in the current optimal solution the hip joint is close to maximum hyper-extension and the gymnast will need to modify further his technique or improve hip strength. Increasing both vertical velocity and angular velocity will improve performance up to point. Higher vertical or angular velocities result in shortened contact times which will decrease the changes in vertical and angular velocity that occur as a result of the contact. Moreover, the shoulder becomes hyper-opened (hyper-flexed) for high vertical velocities and to cope with the increase in velocity the gymnast will need to modify further his technique or improve shoulder strength.

\section{ACKNOWLEDGEMENTS}

The support of British Gymnastics is gratefully acknowledged.

\section{REFERENCES}

Begon, M., Wieber, P-B. Yeadon, M. R., 2008. Kinematics estimation of straddled movements on high bar from a limited number of skin markers using a chain model. Journal of Biomechanics 41, 581-586.

Boone, W. T. 1976. Illustrated handbook of gymnastics, tumbling and trampolining. Parker Publishing Company, Inc. 
Carroll, D.L., 1996. Genetic Algorithms and Optimizing Chemical Oxygen-lodine Lasers. In: Wilson, H.B., Batra, R.C., Bert, C.W., Davis, A.M.J., Schapery, R.A., Stewart, D.S., Swinson, F.F. (eds). Developments in Theoretical and Applied Mechanics. The University of Alabama. 411-424.

Esteki, A., Mansour, J. M., 1996. An experimentally based nonlinear viscoelastic model of joint passive moment. Journal of Biomechanics 29, 443-50.

Fédération Internationale de Gymnastique - FIG. 2013. Code of Points Men's Artistic Gymnastics. http://www.upag-gymnastics.com/en/documentos/magcop13.pdf

Forrester, S. E., Yeadon, M. R., King, M. A., Pain, M. T. G., 2011. Comparing different approaches for determining joint torque parameters from isovelocity dynamometer measurements. Journal of Biomechanics 44, 955-961.

Jackson, M. I., Hiley, M. J., Yeadon, M. R., 2011. A comparison of Coulomb and pseudo-Coulomb friction implementations: Application to the table contact phase of gymnastics vaulting. Journal of Biomechanics 44, 2706-2711.

Hiley, M.J. and Yeadon, M.R. 2003. Optimum technique for generating angular momentum in accelerated backward giant circles prior to a dismount. Journal of Applied Biomechanics 19, 119-130.

Hiley, M. J., Yeadon, M. R., 2008. Optimisation of high bar circling technique for consistent performance of a triple piked somersault dismount. Journal of Biomechanics 41, 1730-1735.

King, M. A., Kong, P. W., Yeadon, M. R., 2009. Determining effective subject-specific strength levels for forward dives using computer simulations of recorded performances, Journal of Biomechanics 42, 2672-2677.

King, M. A. and Yeadon, M. R., 2005. Factors influencing performance in the Hecht vault and implications for modelling. Journal of Biomechanics 38, 145-151.

King, M. A., Yeadon, M. R., Kerwin, D. G., 1999. A two segment simulation model of long horse vaulting. Journal of Sports Sciences 17, 313-324.

Koh, M., Jennings, L., Elliott, B, Lloyd, D., 2003. A predicted optimal performance of the Yurchenko Layout vault in Women's Artistic Gymnastics. Journal of Applied Biomechanics 19, 187-204.

Reiner, R., Edrich, T., 1999. Identification of passive elastic joint moments in the lower extremities. Journal of Biomechanics 32, 539-44.

Smith, T. 1982. Gymnastics: A Mechanical Understanding. Hodder and Stoughton.

Still, C. 1990. BAGA Women's Gymnastics Manual. Springfield Books Limited.

Takei, Y. 1988. Techniques used in performing handspring and salto forward tucked in gymnastic vaulting. International Journal of Sport Biomechanics 4, 260-281.

Takei, Y., 2007. The Roche vault performed by elite gymnasts: Somersaulting technique, deterministic model, and judges' scores. Journal of Applied Biomechanics 23, 1-11.

Takei, Y., Kim, E. J., 1990. Techniques used in performing the handspring and salto forward tucked vault at the 1988 Olympic Games. International Journal of Sport Biomechanics 6, 111-138.

van Soest, A.J., Casius, R.L.J., 2003. The merits of a parallel genetic algorithm in solving hard optimiszation problems. Journal of Biomechanical Engineering 125,141-146. 
Yeadon, M. R. 1990a. The simulation of aerial movement - II. A mathematical inertia model of the human body. Journal of Biomechanics 23, 67-74.

Yeadon, M. R. 1990b. The simulation of aerial movement - I: The determination of orientation angles from film data. Journal of Biomechanics 23, 59-66. 